\title{
ESTRATEGIAS INTERACTIVAS EN LA COMPRENSIÓN DE LECTURAS FILOSÓFICAS DE LOS ESTUDIANTES UNIVERSITARIOS DE AYACUCHO
}

\author{
INTERACTIVE STRATEGIES IN THE UNDERSTANDING OF \\ PHILOSOPHICAL READINGS OF THE AYACUCHO UNIVERSITY \\ STUDENTS
}

\author{
Adolfo Quispe Arroyo ${ }^{*}$, (iD María Flores Gutiérrez ${ }^{1}$, (D) Pedro Huauya Quispe \\ adolfo.quispe@unsch.edu.pe; maria.flores@unsch.edu.pe; pedro.huauya@unsch.edu.pe \\ ${ }^{1}$ Universidad Nacional de San Cristóbal de Huamanga, Ayacucho, Perú
}

*Correspondencia: Adolfo Quispe Arroyo. Email: adolfo.quispe@unsch.edu.pe

Recibido: 29.06.2020 | Aprobado: 20.07.2020

\section{RESUMEN}

El presente trabajo de investigación se realizó con el objetivo de determinar la influencia de las estrategias interactivas en la comprensión de lecturas filosóficas de los estudiantes universitarios de San Cristóbal de Huamanga, Ayacucho. Para tal efecto, nos guiamos en el paradigma positivista y su enfoque cuantitativo en el diseño cuasiexperimental. El área de estudio lo constituyeron los estudiantes universitarios. La muestra estuvo integrada por 30 estudiantes de la Escuela Profesional de Obstetricia que conformaron el grupo experimental y 30 estudiantes de la Escuela Profesional de Enfermería que conformaron el grupo control, ambos grupos de la Facultad de Ciencias de la Salud. Para comparar las calificaciones de los grupos se aplicó prueba de comprensión de lecturas filosóficas. Para el contraste de la hipótesis se empleó la prueba estadística de Wilcoxon. Los resultados de la investigación determinaron que el $70.0 \%$ de los estudiantes del grupo experimental, con la aplicación de las estrategias interactivas se ubicaron dentro del promedio bueno y el $30.0 \%$ se encuentra dentro del promedio excelente, a comparación del grupo control que no alcanzaron dichos promedios. En conclusión, con un nivel de confianza al 95\% y nivel de significancia al 5\%, al obtener un valor $\mathrm{p}<0,05(0,000)$ se afirma que la aplicación de las estrategias interactivas influye significativamente en la comprensión de lecturas filosóficas de los estudiantes universitarios de la Universidad Nacional de San Cristóbal de Huamanga, Ayacucho-Perú. Palabras clave: Estrategias interactivas y comprensión de lecturas filosóficas.

\begin{abstract}
The present research work was carried out with the objective of determining the influence of interactive strategies on the understanding of philosophical readings of university students from San Cristóbal de Huamanga, Ayacucho. To this end, we are guided by the positivist paradigm and its quantitative approach in quasi-experimental design. The study area was made up of university students. The sample consisted of 30 students from the Professional School of Obstetrics that made up the experimental group and 30 students from the Professional School of Nursing that made up the control group, both groups from the Faculty of Health Sciences. To compare the qualifications of the groups, a comprehension test of philosophical readings was applied. To test the hypothesis, the Wilcoxon statistical test was used. The results of the investigation determined that $70.0 \%$ of the students in the experimental group, with the application of interactive strategies, were within the good average and 30.0\% were within the excellent average compared to the control group that did not reach these averages. In conclusion, with a confidence level of $95 \%$ and a significance level of $5 \%$, when obtaining a $p$ value $<0.05(0.000)$, it is stated that the application of interactive strategies significantly influence the understanding of philosophical readings of the University students from the National University of San Cristóbal de Huamanga, Ayacucho-Peru.
\end{abstract}

Keywords: Interactive strategies and understanding of philosophical readings. 


\section{INTRODUCCIÓN}

En la actualidad, el acceso a buena parte del conocimiento continúa a través de los medios escritos, sean por medios físicos o virtuales. Por esta razón, quienes no logran comprender lo que leen en la vida universitaria corren el riesgo de tener problemas a lo largo de su vida, principalmente en la formación de la carrera profesional. Puede que se agrave la situación si se trata de lecturas filosóficas, por la naturaleza, estilos, escuelas y concepciones que ella posee. En la actividad universitaria es imprescindible la comprensión de la lectura en su dimensión profunda, puesto que las diferentes áreas de conocimiento se expresarán en lecturas. Por tanto, el objetivo de la presente investigación fue determinar la influencia de las estrategias interactivas en la comprensión de lecturas filosóficas de los estudiantes universitarios, siendo la interrogante ¿Las estrategias interactivas influirán en la comprensión de lecturas filosóficas de los estudiantes universitarios?

Para la respectiva teoría existen diversos tratados y se tomaron los conceptos fundamentales de interés para el trabajo.

Así, para referirse a la lectura es necesario definir al texto como una composición de signos codificados, a través de un sistema de escritura como el alfabeto, que todos los seres humanos conocemos y utilizamos, regularmente, para comunicarnos, el cual debe tener una unidad de sentido para, primero, ser decodificado y luego entendido por el lector. Por consiguiente, se reconoce la importancia del proceso de codificación en el marco de los textos, siendo los textos filosóficos aquellos que, con las categorías y alegorías propias de la filosofía, brindan mensajes implícitos y explícitos para contextualizar y/o contemporizar, además de los proyectos de interpretaciones y transformaciones del mundo. Un texto filosófico es la visión racional de un filósofo sobre el mundo en forma de reflexión, interpretación y crítica para comprender y transformar.

La lectura filosófica constituye una de las formas de leer contemporáneamente el mundo, más nuevas entre las muchas existentes para el trabajo con la lectura. Su finalidad es que el lector se abra de manera más flexible al mundo y desarrolle una capacidad de escucha mediante el diálogo con el autor a través del texto.

Las lecturas filosóficas están llenas de ironías, apologías, neologismos y de mensajes subliminales cínicos que requiere cierta hermenéutica para su comprensión. Para la comprensión abierta y general se requiere actitudes reflexivas y críticas respecto a la posición de un autor, una corriente o tendencia filosófica en un determinado periodo histórico. Del mismo modo, lo importante en la lectura filosófica es no caer en vicios como: la historiografía, sociologismo y psicologismo del texto. Con estos reduccionismos no podemos tener una mayor lectura del texto y poder abrirnos de manera flexible, porque nos subyugamos a un aspecto del autor.

Una lectura filosófica es desentrañar reflexiva y críticamente lo que el mensaje trasmite, según las posiciones filosóficas de cada autor. No es solamente repetir citas como clichés para adjudicar mecanicistamente al autor. Para Ferreiro (2014), la lectura es una habilidad lingüística de carácter superior, que se vincula, por una parte, a un proceso mental y por otra, se concretiza en la interacción social. Desde esta perspectiva, tanto la lectura como la escritura se consideran como un proceso lingüístico en el cual el 
conocimiento de las probabilidades secuenciales del texto escrito desempeña un papel importante y es también un proceso social y cultural con fines sociales tanto como personales.

La habilidad de la lectura, se constituye de forma activa en los seres humanos, y es un vínculo con los conocimientos que se relaciona con el lenguaje. En la medida que se interactúa con un texto, se construye sentido de lo que se lee, es por ello que el lector es un participante primordial de esta interacción como afirma Jolibert:

Los lectores necesitan poseer habilidades de decodificación, pero también estrategias comprensivas, en el entendido que el lector es un procesador activo del texto, y que la lectura es un proceso constante de emisión y verificación de hipótesis conducentes a la construcción de la comprensión del texto y de control de esta comprensión (2000, p.96).

Solé precisa que: "Asumir el control de la propia lectura, regularla, implica tener un objetivo para ella, así como poder generar hipótesis acerca del contenido que se lee” (1999, p.123). Por las predicciones después de la lectura suponemos lo que puede ocurrir en el texto; gracias a su verificación, a través de los diversos mensajes existentes en el texto podemos dar una interpretación, contemporización y comprensión de lo leído.

Por otro lado, tenemos diferentes estrategias de aprendizaje que tratan de facilitar y optimizar el mismo a través de la comprensión de textos en base a las lecturas. Aunque el uso de categoría estrategia no siempre fue pedagógica. Así Genovard, especifica:

El término estrategia nunca fue un concepto psicológico, ni tan sólo familiar en el campo de las ciencias humanas aplicadas. En realidad, el término estrategia fue inicialmente un término militar que indicaba las actividades necesarias para llevar a cabo un plan previo de operaciones bélicas a gran escala, y el dominio de los diferentes estadios que esto suponía era denominado táctica (1990, p.69).

Por lo referido el término estrategia de orígenes castrenses terminó en el uso constante de acciones pedagógicas buscando el aprendizaje óptimo del estudiante, entre ellas de la lectura. Pressley (1985) afirma que las estrategias de aprendizaje constituyen actividades conscientes e intencionales que guían las acciones a seguir para alcanzar determinadas metas de aprendizaje. Así mismo, Sánchez (1993) refiere que, para entender los textos expositivos, en especial y otros tipos se requieren estrategias para mejorar su comprensión, éstas pueden y deben ser dinámicas y controladas.

Beltrán precisa:

Que un rasgo importante de cualquier estrategia es que está bajo el control del estudiante, es decir, a pesar de que ciertas rutinas pueden ser aprendidas hasta el punto de automatizarse, las estrategias son generalmente deliberadas, planificadas y conscientemente comprometidas en actividades y técnicas (1993, p.143).

Así mismo, Pozo al respecto refiere:

La aplicación de las estrategias de aprendizaje no es automática sino controlada. Precisan planificación y control de la ejecución y están relacionadas con la metacognición o conocimiento sobre los propios procesos mentales. Implican un 
uso selectivo de los propios recursos y capacidades disponibles. Para que un estudiante pueda poner en marcha una estrategia debe disponer de recursos alternativos, entre los que decide utilizar, en función de las demandas de la tarea, aquellos que él cree más adecuados (2000, p.235).

Se observa que los autores coinciden en la elección de las estrategias de aprendizaje de una manera rigurosa, bajo control y dominio del docente, así como del estudiante. La comprensión lectora no sólo depende de la significatividad lógica: cohesión, sintaxis, etc. y psicológica: distancia óptima entre los conocimientos del lector y el contenido del texto. Son imprescindibles también, las estrategias que el lector utiliza para intensificar la comprensión y el recuerdo de lo que lee, así como para detectar y compensar los posibles errores de comprensión. La mentalidad estratégica, precisa Brown "Implica una actitud alerta en la evaluación de la consecución del objetivo, lo cual nos lleva a variar nuestra actuación cuando nos parece necesario" (1988, p.167). Enseñar estrategias es enseñar a aprender a aprender en la dinamicidad de la actividad consciente del quien aprende.

Para leer se requiere habilidades de decodificación y aportar al texto nuestros objetivos, ideas y experiencias previas; necesitamos involucrarnos en un proceso de predicción, inferencia continua y contemporización del mensaje que aporta el texto y en nuestro propio bagaje que permita encontrar o rechazar las predicciones e inferencias. Para Solé (1999), la comprensión del texto requiere estrategias cognitivas y metacognitivas de lectura, pues un carácter metacognitivo, implica conocer el propio conocimiento, capacidad de pensar y planificar la acción. En su calidad de procedimientos elevados que implican lo cognitivo y lo metacognitivo, no pueden abordarse como técnicas rígidas e infalibles, sino como orientaciones para la acción, para representarse los problemas y orientar de forma flexible las posibles soluciones.

Las estrategias interactivas en su calidad de procedimientos elevados implican el trabajo en lo cognitivo, no pueden abordarse como técnicas rígidas e infalibles, menos de disciplinar a manera castrense sino como orientaciones guía para la acción, para representarse los problemas y orientar de forma flexible las soluciones posibles, previa comprensión, interpretación y contemporización. En su condición metacognitiva implica conocer el propio conocimiento, capacidad de pensar y planificar la acción. En definitiva, controlar y regular la acción inteligente de un docente con el estudiante. En la comprensión de la lectura filosófica interviene, no solo el estudiante lector, sino el docente para interactuar constantemente con los estudiantes, sea de manera individual, en pareja o grupal. El docente está en actitud activa e interesada desde el inicio hasta el final de la lectura filosófica, absuelve dudas, dinamiza, motiva y valora las respuestas o interpretaciones de los estudiantes sobre la lectura. Se podría afirmar que el docente lee junto a sus estudiantes y dialogan, de acuerdo a los avances e interpretaciones que van dando forma a la comprensión del texto filosófico. De esta manera, con estrategias interactivas, dinamizamos las lecturas filosóficas.

Por el uso de las estrategias interactivas se renuncia a la visión tradicional del educando como un ser pasivo, para dar paso a un modelo interactivo, en el cual el sujeto asume un papel preponderante al otorgarle un significado a lo que lee y al generar nuevas producciones a partir de lo leído y comprendido, como afirma Colomer y Camps: 
En el modelo interactivo, el lector es considerado como un sujeto activo que utiliza conocimientos de tipo muy variado del escrito y que reconstruye el significado del texto al interpretarlo de acuerdo con sus propios esquemas conceptuales y a partir de su conocimiento previo (2000, p.35).

En este modelo se resalta el papel de lo que aporta el texto, como lo que aporta el lector, lo cual suscita una relación íntima entre uno y el otro. Se podría afirmar que el diálogo autor-lector se concretó bajo la guía del docente. El lector se basa en sus conocimientos para comprender e interpretar el texto y la posición del autor, para extraer un significado y a su vez este nuevo significado le permite crear, contemporizar, modificar, elaborar e integrar nuevos conocimientos en sus esquemas mentales. Por la variedad de las concepciones filosóficas, escuelas, corrientes y disciplinas se requiere mayor atención en la decodificación de los textos filosóficos. La ubicación del tiempo y espacio histórico de las ideas transmitidas es vital para contemporizar los textos filosóficos por la exquisitez y neologismos que cada autor va añadiendo en su discernir filosófico.

Es necesario tomar en cuenta que gran parte de la vida universitaria depende de las habilidades lectoras que el estudiante posee desde la educación básica. De acuerdo con Ramírez (2009), al creer que el gusto por la lectura se adquiere leyendo bajo el efecto de la obligación, para cumplir con una tarea, la escuela deja de lado la posibilidad de que los estudiantes lean por y con placer como debiera presentarse esta actividad intelectual. Las estrategias interactivas se fundamentan en el modelo interactivo de lectura, en el cual el estudiante, es considerado como un ente activo que utiliza diferentes estrategias para obtener la información del texto y construir un nuevo significado, de acuerdo con sus propios sistemas conceptuales desarrollados en su interacción con su medio natural, sociocultural, lingüístico, etc.

La comprensión lectora no sólo depende de la significatividad lógica: coherencia, cohesión, sintaxis y psicológica: distancia óptima entre los conocimientos del lector y el contenido del texto sino también motivación del propio lector. Para Brown (1988), un buen lector es una persona que posee un repertorio de estrategias cognitivas que es capaz de usar de manera flexible durante la lectura. Un lector experto que tiene al menos un conocimiento procedimental sobre estrategias cognitivas más sofisticadas, como sería, por ejemplo, la de emplear más tiempo en estudiar materiales más importantes y menos conocidos que en estudiar los menos importantes y más conocidos.

Las estrategias interactivas que el lector utiliza para intensificar la comprensión y el recuerdo de lo que lee, así como para detectar y compensar los posibles errores de comprensión, implican una actitud de alerta en la evaluación de la consecución del objetivo, lo cual nos lleva a variar nuestra actuación cuando nos parece necesario. Utilizar estrategias interactivas, es enseñar a aprender a aprender. Así para Palincsar y Brown "El aprender a aprender permite al lector seleccionar, emplear, controlar y evaluar el uso de estrategias lectoras" (1997, p.121). Por lo tanto, éstas implican, entre otras cosas, el monitoreo activo y la regulación posterior de las actividades de procesamiento de la información. Solé (1999) afirma que la lectura interactiva persigue los siguientes objetivos: 
- Desarrollar habilidades para construir el significado de los textos, es decir para comprenderlos al anticipar su contenido, formularles preguntas, formar imágenes mentales, comentarlo, resumirlo, etc.

- Desarrollar la lectura crítica en los niños y niñas al estimular el procesamiento de la información de los textos, la conceptualización y la confrontación de sus planteamientos con los de los otros.

- Desarrollar el gusto por la lectura, al generar un ambiente grato, acogedor y afectivo y evidenciar que la lectura puede ser una fuente de aprendizaje y de satisfacción.

- Desarrollar la expresión oral, al promover la interacción sistemática de los niños y niñas alrededor de los textos.

La puesta en práctica de la lectura interactiva, así como el alcance de estos objetivos, permiten formar estudiantes con mayores habilidades de comprensión de lectura en general y filosófica en particular. Requieren a su vez de los docentes un conocimiento riguroso de las estrategias de intervención y que ambos procesos dinámicos, interactivos y aplicables contribuyan a la educación universitaria. A manera de síntesis y solo con fines didácticos podemos sugerir los roles del maestro y del estudiante para cumplir con las estrategias interactivas:

\section{Rol de los estudiantes:}

- De un rol de escucha pasiva al hacer activo generando de las lecturas: discusiones, reflexiones, contemporizaciones, entre otras actividades.

- Se implican en procesos del pensamiento de orden superior tales como: análisis, síntesis, evaluación y contemporización.

- Comprenden la lectura en el diálogo y en la interacción con el contenido y desarrollo de objetivos esperados.

- Los estudiantes son retroalimentados inmediatamente por el docente y por sus compañeros.

Rol de los docentes:

- Presentan las actividades programadas de acuerdo al contenido del plan curricular.

- Aplican la actividad de la lectura a las posibilidades, necesidades y expectativas del grupo.

- Facilitan la actividad de la lectura cuidando la extensión y profundidad del texto filosófico que se aborda.

- Retroalimentan de manera oportuna en las dificultades de la comprensión de la lectura filosófica, siendo esta actividad individual en pares o grupal.

- Persiguen el logro de los objetivos o competencias según lo planificado en la especialidad a tratar.

- Guía y monitorea el diálogo de los estudiantes sobre la lectura.

- Retroalimenta y genera espacios para la coevaluación y autoevaluación.

- Utiliza la tecnología (videos o documentales) para fortalecer la comprensión del texto leído. 
- Congrega las interpretaciones de los estudiantes para profundizar la comprensión del texto filosófico.

- Evalúa de manera formativa la participación de las interacciones en la comprensión de la lectura filosófica.

- Mantiene una actitud empática con apertura al diálogo con los estudiantes.

- Promueve la unidad de la teoría con la práctica en la comprensión de la lectura para contemporizar.

- Genera seguridad y promueve responsabilidad de los estudiantes respecto a las lecturas.

Los resultados de la investigación determinaron que los estudiantes del grupo experimental, con la aplicación de las estrategias interactivas obtuvieron en $70.0 \%$ un promedio de bueno y el 30.0\% lograron el promedio de excelente a comparación del grupo control que no alcanzaron estos promedios. En conclusión, con un nivel de confianza al 95\% y nivel de significancia al 5\%, se afirma que la aplicación de las estrategias interactivas influyó significativamente en la comprensión de lecturas filosóficas de los estudiantes universitarios. Para el contraste de la hipótesis se empleó la prueba estadística de Wilcoxon al 95\% del nivel de confianza.

\section{MATERIALES Y MÉTODOS}

Tipo de estudio: aplicada; nivel de investigación: explicativa; diseño: experimental en su variante cuasi; nivel social, espacial y temporal: estudiantes de la Universidad Nacional de "San Cristóbal de Huamanga" - Ayacucho, 2019; población objetiva: estudiantes universitarios(12 000); muestreo: no probabilístico a criterio del investigador (60) se formaron dos grupos (experimental y de control); criterio de inclusión: estudiantes de la Escuela Profesional de Obstetricia y Enfermería del primer ciclo o serie 100 de la Facultad de Ciencias de la Salud; criterios de exclusión: estudiantes de otras series y otras facultades; método: experimental y comparativo; técnica e instrumento: evaluación/ prueba de lecturas filosóficas; lectura filosófica de la evaluación (alegoría de la caverna de Platón); técnicas de procesamiento de datos: SPSS 2018, paquete estadístico Excel 2014, representado en el cuadro y el gráfico.

\section{RESULTADOS Y DISCUSIÓN}

\section{Tabla 1}

Comprensión de lecturas filosóficas de los universitarios

\begin{tabular}{|c|c|c|c|c|}
\hline \multirow{3}{*}{ CALIFICACIÓN } & \multicolumn{4}{|c|}{ RESULTADOS } \\
\hline & \multicolumn{2}{|c|}{ Grupo Control } & \multicolumn{2}{|c|}{ Grupo experimental } \\
\hline & f1 & $\mathrm{F} \%$ & f1 & F\% \\
\hline Malo $(0-10)$ & 16 & 53.3 & 00 & 0.0 \\
\hline Regular (11-13) & 14 & 46.7 & 00 & 0.0 \\
\hline Bueno (14-16) & 00 & 0.0 & 21 & 70.0 \\
\hline Excelente (17-20) & 00 & 0.0 & 09 & 30.0 \\
\hline Total & 30 & 100 & 30 & 100 \\
\hline
\end{tabular}

Fuente: evaluación a los estudiantes de la Universidad Nacional de San Cristóbal de Huamanga, 2018 


\section{Gráfico 1}

Comprensión de lecturas filosóficas de los universitarios

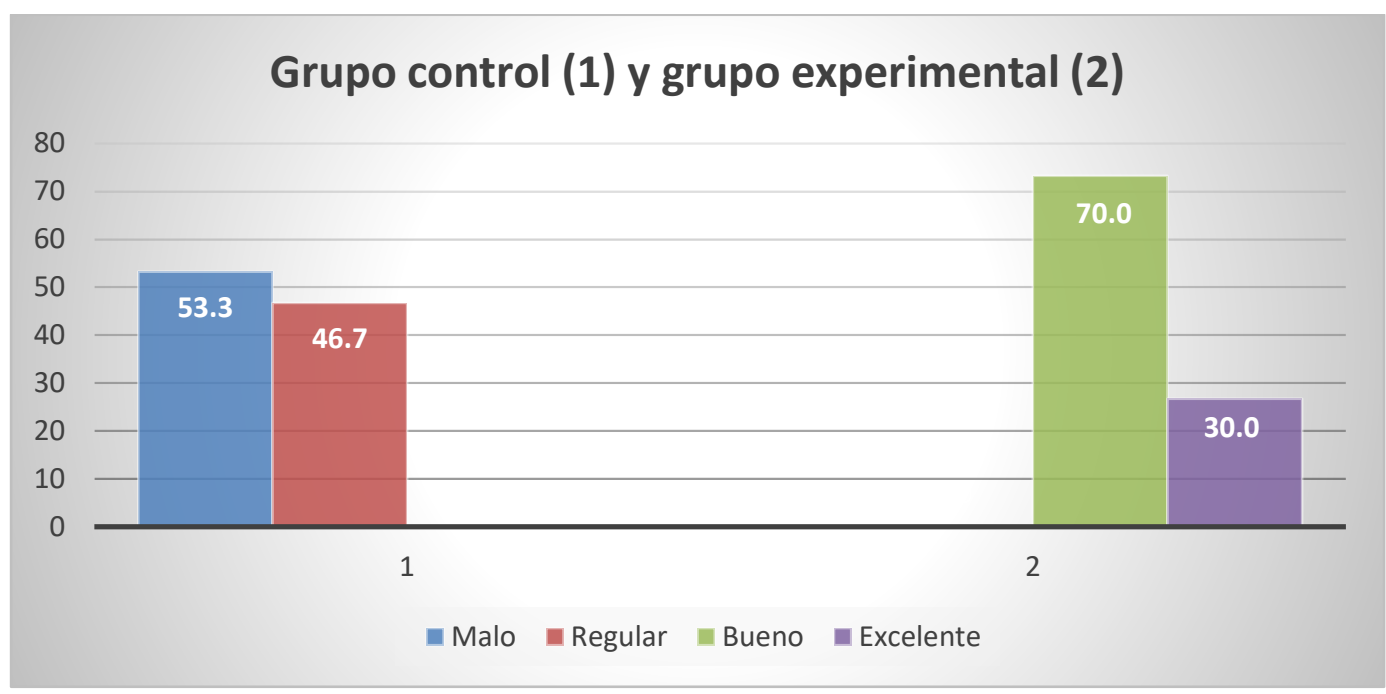

Fuente: evaluación a los estudiantes de la Universidad Nacional de San Cristóbal de Huamanga, 2018

\section{Lectura del cuadro y gráfico}

De los 30 universitarios (100\%), del grupo de control en la comprensión de lecturas filosóficas, se observa que: el 53.3\% (16) se ubican dentro del promedio malo, el 46.7\% (14) está en promedio regular, ninguno se ubica en el promedio bueno y excelente. Por otro lado, de los 30 estudiantes universitarios (100\%), del grupo experimental, se observa que: ningún estudiante universitario se ubica dentro del promedio malo ni en regular y el $70.0 \%$ (21) se encuentran dentro del promedio bueno, y 30.0\% (9) se ubican dentro del promedio excelente.

En la comparación de promedios entre los grupos experimental y control sobre la comprensión de lecturas filosóficas de los universitarios se evidenció preponderancia del promedio del grupo experimental sobre el grupo control, detectada mediante la prueba de Willcoxon, al obtener $\mathrm{p}<0,05(0,000)$ con nivel de significancia al 5\% rechazando la hipótesis nula y aceptando la hipótesis de investigación. En consecuencia, se acepta que las estrategias interactivas influyen significativamente en la comprensión de lecturas filosóficas en los estudiantes universitarios (Grupo experimental respecto al grupo control). Por tanto, se sostiene que existen diferencias significativas en la comprensión de lecturas filosóficas entre el grupo experimental con aplicación de estrategias interactivas y el grupo control sin aplicación de dicha estrategia.

Por el uso de las estrategias interactivas se renuncia a la visión tradicional del educando como un ser pasivo, para dar paso a un modelo interactivo, en el cual el sujeto asume un papel preponderante al otorgarle un significado a lo que lee y al generar nuevas producciones a partir de lo leído y comprendido, para luego confrontar con la realidad natural, social y pensamiento. Lo importante en la lectura filosófica es no caer en vicios como la historiografía, sociologismos y psicologismos del texto, porque con estas parcelaciones no podemos tener una mayor lectura del texto filosófico de manera flexible a las ideas principales, porque nos reducimos a un aspecto minúsculo del autor. Con las estrategias interactivas se minimizan los vicios improductivos o mecanicistas en una 
lectura y se hace viva la actividad en atención y mirada del docente al estudiante lector. Lo filosófico implica digerir reflexiva y críticamente lo que el mensaje transmite, según las posiciones filosóficas de cada autor con su invitación al diálogo prudente o subliminal que podría generar dificultades en la comprensión del lector; sin embargo, con la mirada y participación vigilante del docente se hará fácil e interesante la lectura, puesto que implica confianza, conocimiento y guía para la lectura previamente seleccionada por el educador. De esta manera, se concretiza la interactividad entre el docente guía y el estudiante lector.

Por los resultados solo se reafirma y se avala otras investigaciones de influencia de las estrategias interactivas en el proceso de aprendizaje y de las lecturas de estudiantes de Educación Básica Regular, Técnica Superior y Superior Universitaria, indistintamente. Por ello, se considera innecesaria la discusión teórica del caso, puesto que revalidamos las investigaciones de otros autores por los resultados parecidos.

\section{CONCLUSIÓN}

Las estrategias interactivas se fundamentan en el modelo interactivo de lectura, en el cual el estudiante es considerado como un ente activo que utiliza diferentes estrategias para obtener la información del texto y construir un nuevo significado, de acuerdo con sus propios sistemas conceptuales desarrollados en su interacción con su medio natural, sociocultural, lingüístico, etc. En especial, por el diálogo alcanzado entre el lector y el autor bajo la guía del docente.

En la presente investigación se consideró las estrategias interactivas en la comprensión de las lecturas filosóficas de los estudiantes universitarios formados en dos grupos, cuyo resultado establece diferencias significativas en la comprensión de lecturas filosóficas, entre el grupo experimental de estudiantes con estrategias interactivas y el grupo control que no aplica dicha estrategia.

Existen diferencias significativas en la comprensión de lecturas filosóficas entre el grupo experimental de estudiantes con estrategias interactivas y el grupo de control que no aplica dicha estrategia en la Facultad de Ciencias de la Salud de la Universidad Nacional de "San Cristóbal de Huamanga". Esta afirmación se basa en la prueba estadística de Wilcoxon con un nivel de confianza al 95\% y nivel de significancia al 5\%, al obtener un valor $\mathrm{p}<0,05(0,000)$.

Finalmente, se invoca a la comunidad científica a continuar con las investigaciones de los beneficios de la aplicación de las estrategias interactivas en la comprensión de lecturas filosóficas o de otras áreas del conocimiento. Así como, sus estudios desde diferentes enfoques y paradigmas para validar y revalidar la influencia de las estrategias interactivas en la comprensión de lecturas filosóficas para ser generalizadas en condiciones parecidas y considerarlas dentro de las estrategias a utilizar en la educación universitaria.

\section{REFERENCIAS BIBLIOGRÁFICAS}

Beltrán, J. (1993). Procesos, estrategias y técnicas de aprendizaje. Madrid: Síntesis.

Brown A. (1988) Metacognition, Executive Control, Self-regulation, and other More Mysterious. Broadway: Eds. 
Colomer y Camps (2000) Enseñar a leer, enseñar a comprender. España: Celeste. Ediciones/ mec.

Ferreiro, E. (2014) El ingreso a la escritura y a las culturas de lo escrito. España: Siglo XXI.

Genovard, C. (1990) Psicología de la instrucción. Lima: Santillana.

Jolibert, J (2000) Lo que es leer. México: Mc Graw-Hill.

Palincsar y Brown (1997) La enseñanza para la lectura autorregulada. En L. Resnick y L.

Kopfler (comps.) Broadway: Currículum y cognición.

Pozo, J. (2000) Adquisición de estrategias de aprendizaje. Madrid: Cuadernos de pedagogía.

Pressley, K. (1985) Tite relevance of tite goad stralegy ¿ser niodel (o lite teaching of rrmathero.alics. Educational Psycrdagíst. N.Y: Wiley-

Ramírez, E. (2009) ¿Qué es leer? ¿Qué es la lectura? Centro Universitario de Investigaciones Bibliotecológicas. México: UNAM-

Sánchez, M. (1993) Los textos expositivos. Estrategias para mejorar su comprensión. Lima: Santillana.

Solé, I. (1999) Estrategias de lectura. Barcelona: Grao.

\section{CITAR COMO:}

Quispe Arroyo, A., Flores Gutiérrez, M., \& Huauya Quispe, P. (2020). Estrategias interactivas en la comprensión de lecturas filosóficas de los estudiantes universitarios de Ayacucho. Puriq, 2(3), 186-195. https://doi.org/10.37073/puriq.2.3.93 